\title{
APRENDIZAGEM ADAPTATIVA ONLINE: UMA EXPERIÊNCIA USANDO TRILHAS E CHATBOT PARA DESENVOLVER COMPETÊNCIAS BÁSICAS EM LÍNGUA PORTUGUESA E MATEMÁTICA PARA O ENSINO SUPERIOR
}

\section{ADAPTIVE ONLINE LEARNING: AN EXPERIENCE USING PATHS AND CHATBOT TO DEVELOP BASIC COMPETENCIES IN PORTUGUESE LANGUAGE AND MATHEMATICS FOR HIGHER EDUCATION}

\author{
Marcos Andrei Ota \\ Universidade Cidade de São Paulo,marcos.ota@cruzeirodosul.edu.br
}

\section{Sara Dias-Trindade,}

Universidade de Coimbra, sara.trindade@uc.pt

Carlos Fernando Araujo Júnior

Universidade Cruzeiro do Sul, carlos.araujo@cruzeirodosul.edu.br

Alberto Messias da Costa Souza

Universidade Cruzeiro do Sul, alberto.souza@cruzeirodosul.edu.br

\section{Resumo}

Este trabalho apresenta uma proposta de sistema de aprendizagem adaptativa online piloto, estruturado por meio trilhas de aprendizagem e integração de chatbot para subsidiar o desenvolvimento de competências básicas dos estudantes ingressantes em cursos superiores. O sistema online adaptativo foi utilizado para o desenvolvimento de dois cursos online com o objetivo de promover as competências básicas de aprendizagem em Matemática Básica e Língua Portuguesa. O estudo recorreu à metodologia de pesquisa design-based research que se caracteriza, entre outros aspectos, por ciclos de iterações e contínuas melhorias dos artefatos ou sistemas desenvolvidos. Neste artigo apresentamos a fundamentação da literatura e o compartilhamento das etapas de desenvolvimento do sistema proposto e de sua aplicação em 02 cursos de extensão online em uma instituição de ensino superior brasileira. Um total de 10 mil estudantes foram impactados com a formação. Dos resultados obtidos até aqui, destaca-se que com a importância da metodologia de design-based research para o desenvolvimento do sistema adaptativo piloto e suas sucessivas melhorias e que a integração de um assistente virtual, amplia-se a possibilidade de personalização do ensino. Relativamente aos resultados, pode-se dizer que o sucesso obtido com o projeto piloto se deve muito às possibilidades advindas da nova geração de ambientes virtuais, como por exemplo, a combinação e integração de tecnologias digitais que possam se aproximar das necessidades específicas dos estudantes; formas de avaliar e dar feedbacks durante o processo da aprendizagem. De 
modo geral, as estratégias adaptativas adotadas representam uma contribuição para o ensino, na melhoria dos indicadores de permanência do estudante, além de potencializar a experiência do usuário e permitir a recomendação de conteúdos.

Palavras-chave: Sistemas Adaptativos, Personalização da Aprendizagem Trilhas de Aprendizagem; Educação a Distância; Educação Superior;

\section{Abstract}

This paper presents a proposal for a pilot project of an adaptive learning system, structured through the learning paths and chatbot integration to subsidize the development of basic skills of incoming students in higher education. The adaptive online system was used for the improvement of two online courses with the purpose to boost learning skills in Basic Math and Portuguese. This study was based on the research design-based methodology that is characterized, among other aspects, by cycles of iterations and continuous improvements of the artifacts or systems developed. In this article, we present the fundaments of the literature and the sharing of the stages of development of the proposed system and its application in 02 online extension courses in a Brazilian higher education institution. With a total of 10,000 students were impacted by the training. From the results obtained so far, it is worth noting that with the importance of the design-based research methodology for the development of the adaptive system and its successive improvements, and that the integration of a virtual assistant increases the possibility of personalization of teaching. About the results, it can be said that the success obtained of the pilot project is due to the possibilities of the new generation of virtual learning systems, such as the combination and integration of digital technologies that can approach the specific needs of students; ways of evaluating and giving feedback during the learning process. In general, the adaptive strategies adopted represent a contribution to teaching, improving student permanence indicators, as well as enhancing the user experience and allowing the recommendation of contents.

Keywords: Adaptive Systems, Personalization of Learning; Learning path ; Distance Education; High Education;

\section{Introdução}

As Novas Tecnologias Digitais de Informação e Comunicação têm contribuído para a massificação da educação, por exemplo, nos sistemas de educação online ou híbrido, existentes no ensino superior no Brasil e no mundo. Contudo, os mais recentes avanços têm possibilitado a personalização da aprendizagem por meio dos chamados sistemas adaptativos de aprendizagem.

Os sistemas adaptativos de aprendizagem, em linhas gerais, são sistemas que possibilitam a personalização da aprendizagem orientado por um ou mais fatores ou recursos como: i) uso de algoritmos específicos, considerando os aspectos de learning analytics para recomendações de percursos e conteúdos diferenciados; ii) mecanismos de avaliação que permitem a remediação; iii) resultados de avaliação para propor caminhos 
de aprendizagem programados; iv) escolha e interação do estudante, baseado em características pessoais para então, desenvolver percursos diferenciados de aprendizados.

De acordo com o Plano Nacional de Educação dos Estados Unidos de 2017, o aprendizado personalizado é definido como " instrução na qual o ritmo de aprendizado e as abordagens de aprendizagem são otimizadas pela contemplação das necessidades de cada aprendiz. Neste contexto, os objetivos de aprendizagem, os métodos instrucionais, os conteúdos (e seu sequenciamento) podem todos variar e assumem percursos distintos. As atividades de aprendizagem, portanto, são significativas e relevantes para os aprendizes, direcionadas pelos seus interesses e, frequentemente, pela sua autoiniciativa" (U. S. Department of Education, 2017, p. 9).

Embora o uso dos termos personalização da aprendizagem e sistemas adaptativos tenham sido utilizados de forma a caracterizar o mesmo assunto, há diferenças apontadas por alguns autores. Enquanto as abordagens para a personalização da aprendizagem possam ser diversas, usando ou não tecnologia, os sistemas adaptativos de aprendizagem têm sido associados com tecnologia que se utilizam de recursos e funcionalidades para a personalização da aprendizagem (XIE et al, 2019).

Uma associação possível, ainda, na proposta de ambientes online de aprendizagem adaptativas é o uso de estilos de aprendizagem (TRUONG, 2016). Os estilos de aprendizagem podem ser caracterizados como preferências e ou tendências pessoais do estudante para aprender. Keefe (1979) caracteriza estilos de aprendizagem como a composição de características cognitivas, afetivas, e fatores psicológicos que serve como indicador relativamente estável de como o aprendiz percebe, interage e responde ao ambiente de aprendizagem.

Neste trabalho apresentamos uma proposta de um sistema de aprendizagem adaptativa online piloto, concebido a partir de recursos computacionais de um ambiente virtual (Blackboard Learn), que permite desenvolver trilhas de aprendizagem diferenciadas para os estudantes, a partir de regras e condições programáveis específicas (avaliação, resultados de avaliação ou escolha própria do estudante). A proposta foi utilizada para o desenvolvimento de dois cursos com trilhas de aprendizagem adaptativas com objetivo de proporcionar as competências básicas na área de Matemática (CBL-MAT) e Língua Portuguesa (CBL-LPO). Para apoio dar suporte às dúvidas dos alunos, foi implementado também um chatbot. A metodologia de pesquisa utilizada foi a Design-based Research (REEVES, 2006; ANDERSON, SHATTUCK, 2012).

\section{Trilhas de aprendizagem adaptativas e a personalização do ensino}

Do ponto de vista pedagógico, pode-se dizer que modelos de aprendizagem com foco no estudante possuem essencialmente características advinda do ensino personalizado e da aprendizagem baseada em competências. Ter um ambiente personalizado, no entanto, requer um caminho, que, na literatura, é denominado trilha de aprendizagem, são referenciadas também como: learning path e/ou percurso de aprendizagem. Dito de outra forma, as trilhas de aprendizagens são abordadas sob dois pontos de vista (RAMOS \& OLIVEIRA, 2015): 1. sequência de conteúdo (materiais didáticos, recurso educacional, atividade, fórum, chat, etc) definida pelo professor, ao 
planejar a disciplina, e disponibilizada no ambiente virtual (AVA); ou 2. Caminho percorrido pelo estudante, durante a sua interação com recursos disponibilizados no ambiente virtual.

As trilhas de aprendizagem correspondem a "caminhos virtuais de aprendizagem, capazes de promover e desenvolver competências no que concerne ao conhecimento, à habilidade, à atitude, à interação, à interatividade e à autonomia" (TAFNER et al, 2012, p. 5). Nesse sentido, por meio da orientação direta, proporciona a flexibilização do percurso necessário para se atingir os objetivos de aprendizagem.

Três abordagens principais para a personalização do ensino são consideradas na visão de Graf et al. (2012, p.20): a filtragem baseada em conteúdo, a filtragem colaborativa e técnicas de filtragem baseadas em regras. De acordo com o autor, essas técnicas são diferenciadas pelo tipo de dados de entrada coletados para criar perfis de usuários, pelas estratégias usadas para construir esses perfis e pelo método usado para fazer previsões ou fornecer conteúdo personalizado.

Na perspectiva de Brusilovsky (1994), a disponibilização de conteúdo personalizado para estudante possui quatro módulos possíveis:

- Módulo Aluno - responsável pela construção e atualização do modelo do aluno, inclui todas as informações relevantes que o sistema reuniu sobre o aprendiz.

- Módulo Especialista - responsável pelo conhecimento do domínio (por exemplo, os fatos e regras de um domínio específico), que é armazenado no modelo especializado e para a representação interna do conhecimento de domínio no sistema.

- Módulo de Tutoria - fornece informações sobre como o material de aprendizagem, disponível no modelo especialista, pode ser apresentado de forma adequada, considerando as necessidades individuais do aluno, acessadas através do modelo estudantil.

- Módulo de Interface - responsável por apresentar o conteúdo determinado pelo módulo de tutoria e controla a comunicação e a interação dos alunos com o sistema.

Ancorado às ideias suscitadas até aqui, ao relacionar as estratégias pedagógicas com as inúmeras possibilidades de desenvolvimento de cursos personalizados e adaptativos, tem-se um cenário promissor com o surgimento de uma nova geração de ambientes virtuais que permite a integração de recursos, a aplicação de estratégias adaptativas em conteúdos e/ou cursos, baseando-se pelas ferramentas e novas funcionalidades para incorporar trilhas de aprendizagem seja por meio da personalização 
em nível de apresentação de conteúdo ou em nível de navegação, que orienta as pessoas a encontrarem o conteúdo, sugerindo caminhos personalizados.

Dentre as principais possibilidades inovadoras, tem-se: recursos para monitoramento do desempenho e metas, regras adaptáveis para personalização da aprendizagem, elementos de gamificação, portfólio, fórum, modelos de atividades em grupos ou revisão por pares, etc. Outro fator importante refere-se ao fato desses AVAs permitirem facilmente a integração de softwares, conteúdo SCORM, serviços e outras aplicações via API. A saber, O Blackboard, ambiente virtual escolhido para desenvolver a trilha de aprendizagem, possui alguns recursos para personalizar a aprendizagem, além de incluir funcionalidades advindas dos sistemas adaptativos e/ou permitir a integração de aplicações externas.

Ademais, uma matéria publicada por Katsifli (2017) apresenta duas maneiras de pensar sobre a aprendizagem adaptativa enquanto estratégia para desenvolver trilhas de aprendizagem no AVA: (1) quando os recursos para adaptar quais atividades ou conteúdo (e quando) são apresentados a um aluno com base em determinadas características do aluno são construídos dentro do ambiente de aprendizagem. Neste caso, o ambiente de aprendizagem controla a adaptatividade do ensino e da aprendizagem e (2) quando os alunos podem escolher suas atividades de aprendizagem e seu sequenciamento com base em preferências pessoais. Aqui, o aprendiz está no controle da adaptatividade da aprendizagem.

Como exemplo de personalização a ser alcançada no ambiente virtual: conhecimento prévio; nível de engajamento no curso e desempenho medido através de testes ou resultados de cursos. Esses fatores podem ser usados para pré-programar os tipos e a sequência de atividades de aprendizagem. A programação pode ser suficientemente sofisticada para detectar mudanças no comportamento dos alunos ao longo do caminho e oferecer diferentes caminhos de aprendizagem.

Além disso, pode-se ainda adicionar ferramentas para que os alunos continuem a personalizar sua aprendizagem, como selecionar uma variedade de maneiras de estudar, por exemplo, usando navegação linear através de materiais de curso ou usando uma abordagem de conceitos relacionados. Ou a capacidade de escolher com quem eles trabalham em seu grupo de pares e como, onde e quando eles colaboram. É aqui que a tecnologia pode definitivamente ajudar a capacitar os alunos para personalizar sua aprendizagem. Um outro ponto de destaque no desenvolvimento de trilhas, referem-se aos textos instrucionais, às questões interacionais e as dinâmicas de feedback para as atividades avaliatórias.

Com relação aos cursos híbridos, é interessante notar a vantagem em se adotar o passo a passo acerca da personalização. Graças ao perfil não-presencial que o modelo compreende, o aluno é livre para criar relações com os conteúdos que melhor suprem suas necessidades e, assim, pode moldar seu próprio ensino, proporcionando um aprendizado personalizado em sua própria trilha. Com isso, a identificação dos recursos e ferramentas disponíveis no AVA, configura-se como a primeira etapa para o desenvolvimento das estratégias adaptativas. 
Para contextualizar o leitor, a estrutura do modelo de ambiente virtual proposto levou em consideração a revisão da literatura a fim de realizar o planejamento da trilha de aprendizagem, associando aos objetivos de aprendizagem, às regras adaptáveis e às configurações do AVA, no sentido de possibilitar a personalização à medida que os conteúdos disponibilizados fossem aprendidos e/ou superados.

\section{Chatbot em ambientes virtuais}

Os avanços tecnológicos empenhados para o aprimoramento dos recursos e melhorias na experiência dos usuários, fazem dos ambientes virtuais um espaço de ensino e aprendizagem repleto de possibilidades. A integração de diferentes mídias, linguagens, objetos de aprendizagem e as várias formas de promover interação entre alunos, professores e objetos de estudos são ainda pontos fortes a se destacar.

Guiados pelos princípios heutagógicos, muitos modelos institucionais ainda esbarram em algumas limitações técnicas e dificuldades de acesso e/ou navegação apresentadas pelos estudantes no ambiente do curso escolhido. Neste sentido, nota-se também iniciativas de alguns trabalhos (PRIMO et al, 2000; SGANDERLA et al, 2003; REATEGUI e LORENZATTI, 2005; SANTOS, 2009) que buscaram incorporar no ambiente virtual, assistentes virtuais na tentativa de minimizar os problemas apresentados pelos estudantes durante o percurso do curso escolhido. Fator esse que tende a se agravar quando o modelo do curso não prevê a presença do tutor, sendo apenas concebido de forma autoinstrucional.

No campo da inteligência artificial, houve um esforço considerável de pesquisas que buscaram formas de simular a comunicação humana. Os chatbots ou assistentes virtuais caracterizam-se por sistemas desenvolvidos para simular a conversa humana, tendo como principal objetivo promover interação por meio de perguntas e respostas, criando a sensação no usuário de estar conversando com outra pessoa. Rist et al (2003) classificou em dois níveis a interatividade promovida entre chatbots e os usuários. O primeiro nível apresenta apenas as informações aos usuários, sem promover muito a interatividade. Já o segundo nível, promove a interação com os usuários a partir da utilização de diálogos em linguagem natural.

Muito embora o uso chatbots não tenham suas raízes no campo educacional, notase que há um esforço considerável entre as instituições de ensino em explorar alternativas de atendimento em seus sistemas educacionais; principalmente, iniciativas para integração no ambiente virtual com a finalidade de otimizar a troca de informações entre docentes e estudantes para aprimorar o processo de ensino e aprendizagem, configurando-se como um assistente virtual para apoiar questões técnicas, informações de navegação, da disciplina ou do curso e principais dúvidas mapeadas. Um ponto favorável a adição desse recurso está em possibilitar que a experiência do estudante tenha uma relação mais humana durante a interação nos AVAs, ao simular aspectos da presencia social (GARRISON \& ANDERSON, 2003).

Tibola et al (2014) reforçam a ideia de que um aluno pode ficar frustrado se a falta de suporte adequado evitar a conclusão bem-sucedida de uma tarefa, sendo portanto, aconselhável fornecer-Ihe algum complemento do monitor humano para orientar, ajudar e 
dar feedback. Fonte et al (2009) destacam também a capacidade dos assistentes virtuais resolver dúvidas como um tutor real, possibilitando até estabelecer uma conversa social ou sobre a recomendação de um conteúdo específico. Na próxima seção, o leitor poderá conhecer o assistente virtual (Zeki) desenvolvido pelo núcleo de educação a distância (UNIPÊ).

\section{Competências Básicas de Aprendizagem no Ensino Superior}

A evasão é, certamente, um ponto de preocupação e objeto de investigação de muitas instituições de ensino. Diante da expansão da oferta e procura por cursos em nível superior, os fatores de abandono estudantil representam perdas significativas aos setores público e privado, não apenas pelos indicadores acadêmicos, mas também de ordem econômica e social.

As principais causas da evasão (do curso, da instituição e/ou do sistema) no ensino superior, estão relacionados à inadaptação do ingressante ao estilo universitário, falta de maturidade e formação básica deficiente são determinantes para o abandono do sistema, criando dificuldades de adaptação e acompanhamento do curso.

Com o aumento acelerado de cursos a distância, o Ministério da Educação (MEC) brasileiro tem intensificado diretrizes para reduzir a evasão nas universidades; a reforma do ensino médio, surge como uma primeira ação para melhorar esses indicadores, por exemplo. A saber, desde 2015, após a divulgação do Censo da Educação Superior com o perfil dos estudantes matriculados nos cursos de graduação, os indicadores revelaram um aumento considerável no percentual de estudantes que abandonaram os cursos para os quais foram admitidos.

O novo Decreto o 9.057 publicado em 2017, regulamentando a EaD no país, as instituições se veem obrigadas a investirem em tecnologia e ampliar as exigências no preparo das equipes envolvidas e, consequentemente, buscar soluções para ampliar as possibilidades de oferta de cursos, melhorias didático-pedagógicas, diminuir evasão, atender às exigências do MEC com a criação de projetos/programas institucionais para subsidiar competências básicas com nivelamento em Matemática e Língua Portuguesa, promover modelos educacionais que caminhem na mesma direção das transformações e exigências de mercado, como por exemplo: o ensino híbrido, difundido também pelo termo blended learning ou b-learning.

Um ponto importante para assegurar a permanência dos estudantes frente à massificação de ensino, num descompasso entre a qualidade de ensino básico e o que se espera para o acompanhamento adequado de cursos em nível superior, está em impulsionar ações pedagógicas apoiadas pelas tecnologias digitais que forneçam condições mínimas aos alunos para subsidiar o acompanhamento das disciplinas. Ancorado a esta ideia, os estudos de Nicolete (2016) apontam que as atividades interdisciplinares apoiadas no desenvolvimento de competências sugerem uma transformação na forma de ensinar, estimulando a conexão entre os saberes, explorando a experimentação e a prática, colaborando para que os processos de ensino e aprendizagem sejam mais atrativos e motivadores para estimular 0 ingresso e a permanência dos estudantes nessas áreas. 
Em esclarecimento ao leitor, o termo "competências básicas de aprendizagem" atribuído aos cursos deste artigo foi utilizado em oposição ao conceito de "nivelamento", apresentado pelo Ministério da Educação, como exigência e recomendação às instituições de ensino superior para atividades de atendimento aos alunos, com programas de nivelamento e reforço pedagógico. Tratando-se de aprendizagem, acredita-se que não se pode "igualar" ou "nivelar" o conhecimento de um estudante ao outro, até mesmo de uma turma a outra; no entanto, oportunizar situações em que se possa desenvolver competências básicas de aprendizagem (Língua Portuguesa e Matemática, por exemplo) seja mais coerente em razão de diferentes contextos e necessidades de aprendizagem dos estudantes de cursos superiores presenciais e/ou a distância.

$\mathrm{Na}$ seção seguinte, tem-se no modelo de trilha de aprendizagem, uma ação interventiva para desenvolver competências básicas a partir de estratégias adaptativas e integrações de ferramentas tecnológicas para personalizar a aprendizagem dos alunos.

\section{Percurso metodológico}

Sob o ponto de vista metodológico esta pesquisa foi desenvolvida utilizando-se o framework do design-based research (REEVES, 2006 ; ANDERSON, SHATTUCK, 2012). Além do estudo da literatura, consulta de experts nos temas correlatos a pesquisa. $O$ desenvolvimento da proposta de sistema de aprendizagem adapativo online basedo no desenho da trilha de aprendizagem foi estruturado em três etapas : 1. projeto piloto para validação 2. consolidação do modelo e 3. aprimoramento da oferta dos cursos.

A etapa piloto compreendeu o entendimento das dificuldades de aprendizagem apresentadas pelos estudantes ingressantes nos cursos superiores a distância. Cerca de 180 estudantes de cursos híbridos das Engenharias colaboraram para a validação do modelo. Nesse sentido, a partir da seleção do corpo docente (21 professores) e do mapeamento das principais dificuldades, pensou-se na elaboração de dois cursos online e autodirigidos, isto é, sem tutoria, que pudessem responder o desafio de subsidiar as necessidades trazidas pelos alunos que em suma, reveleram fragilidades de pré-requisitos advindas da educação básica, para acompanhar disciplinas complexas que exigiam um conhecimento prévio de Matemática e de Língua Portuguesa.

Para a elaboração de ambos os cursos (Competências Básicas de Matemática e Língua Portuguesa - CBL MAT / LPO), alguns recursos nativos do ambiente virtual foram utilizados para criar trilhas de aprendizagem, sendo estes: estratégias adaptativas (Adaptative Release) para liberação e recomendação de conteúdos, definição dos objetivos e competências (Goals), elementos da gamificação (Achievements) e definição de rubricas/critérios para a ferramenta avalição por pares (Peers Review).

$O$ desenho da trilha de aprendizagem que fora validada pelos estudantes. No modelo em questão (Figura 1), o aluno tem acesso aos conteúdos e acompanhar o seu desempenho de forma personalizada. $\mathrm{O}$ uso do recurso Adaptive Release representa as regras utilizadas para verificar as competências básicas dos estudantes, baseando-se nos desempenhos e metas alinhadas de forma granular aos tópicos de aprendizagem, pertencentes a cada competência macro. 
Após o teste de verificação, o estudante recebe um plano de estudo, conforme o desempenho apresentado e a cada objeto de aprendizagem concluído, são lançados desafios, ancorando-se pelos princípios de gamificação para avaliar a competência estudada. Nesse ponto, as atividades em formato de "Desafio" são também estratégias adaptativas, uma vez que ao apresentar dificuldades para superar uma atividade, são propostos materiais complementares para subsidiar, de forma personalizada, o entendimento do conteúdo. O encerramento do curso está condicionado a um desafio final com atividades de acordo com o perfil de aprendizagem. Por fim, o certificado do curso é disponibilizado aos estudantes, permitindo a integração de certificação à rede social Linkedin e a comprovação de atividade complementar acadêmica.

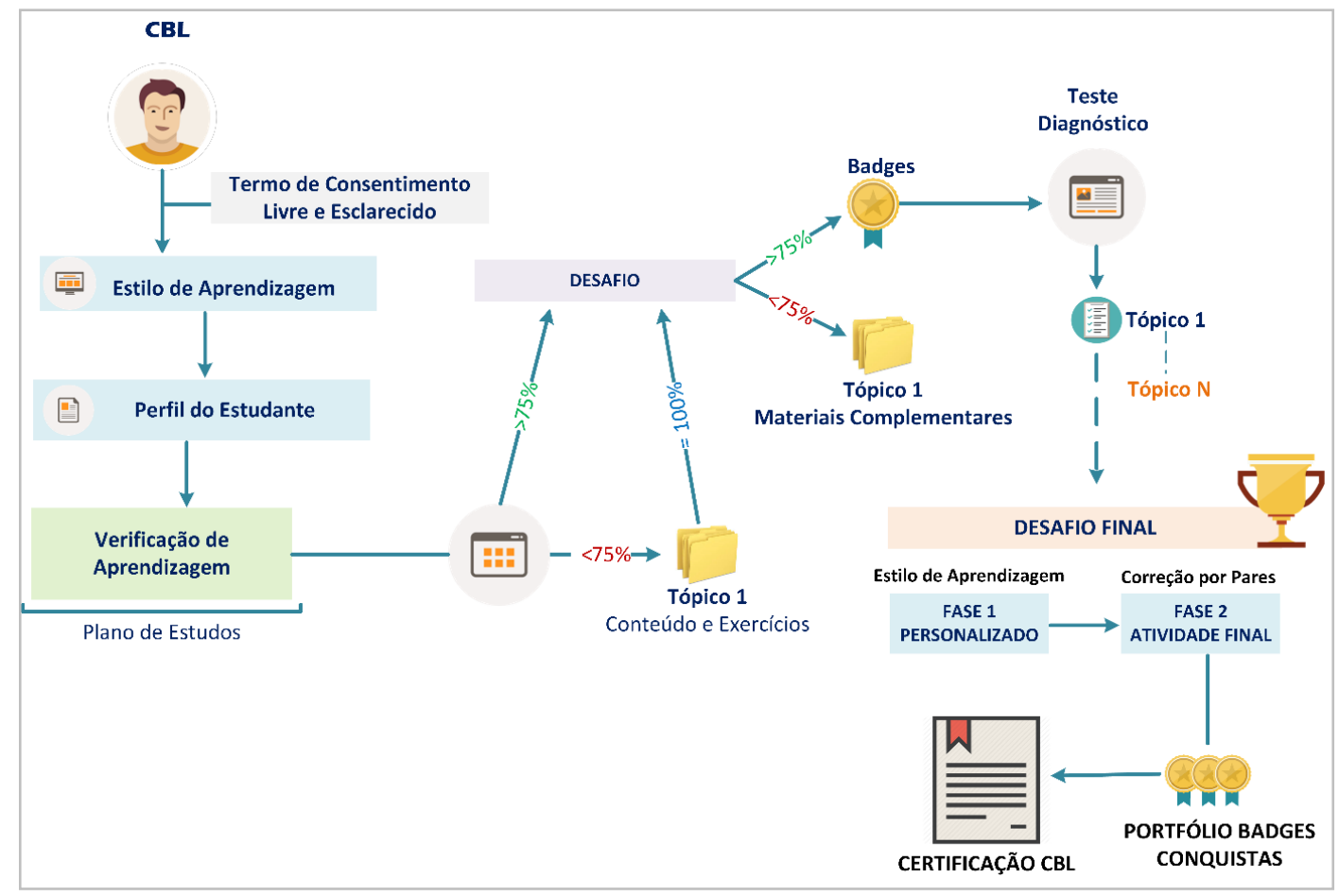

Figura 1. Desenho da Trilha de Aprendizagem Fonte: Ota (2018)

A experiência do estudante após a definição do plano de estudo foi ancorada nas contribuições dos princípios de learning design (LD), que se referem a uma variedade de formas de conceber experiências de aprendizagem dos alunos, ou seja, uma sequência de tipos de atividades e interações. O LD pode estar no nível de um assunto, ou componentes do assunto. Segundo Oliver (1999), a aplicação do desenho da aprendizagem está apoiada nos seguintes elementos-chave: (i) tarefas que os alunos devem fazer; (ii) recursos que ajudam os alunos a realizar a tarefa e (iii) mecanismos de suporte desenvolvidas por um professor.

O aprimoramento dos conteúdos e suas respectivas atividades teve como apoio, o feedback dos próprios estudantes na conclusão de cada competência macro (CM). As estratégias adaptativas, contempladas nos cursos (CBL MAT e LPO), possibilitaram o aluno ter uma noção exata das competências a serem desenvolvidas, tratando-se de modelos de 
cursos híbridos, por exemplo, há uma contribuição importante, pois funcionam como mecanismos de apoio, oportunizando um melhor acompanhamento das disciplinas ofertadas no formato online e ainda potencializar o engajamento e participação dos aprendizes nos encontros presenciais.

Baseado nas contribuições dos alunos (turmas Engenharias), a etapa 2 de consolidação do modelo (Figura 2), foi ajustada a partir das seguintes dimensões: conteúdo, avaliação da aprendizagem, aspecto pedagógico, aspecto social e profissional, elementos técnicos e usabilidade (OTA, 2018). Para este momento, o curso CBL MAT foi ofertado aos novos alunos dos cursos das Engenharias (Ambiental, Produção, Computação, Mecânica, Elétrica e Civil) sendo estes, ingressantes do segundo semestre (2018) em formato blearning. Já o CBL LPO teve a participação dos estudantes dos cursos a distância de Relações Públicas e Publicidade e Propaganda. Cerca de 3000 mil estudantes participaram da formação com duração prevista de 60 dias. Ainda nesta etapa, ambos os cursos tiveram o monitoramento de tutores para dar suporte às questões técnicas e de ordem pedagógica acerca da trilha de aprendizagem consolidada.

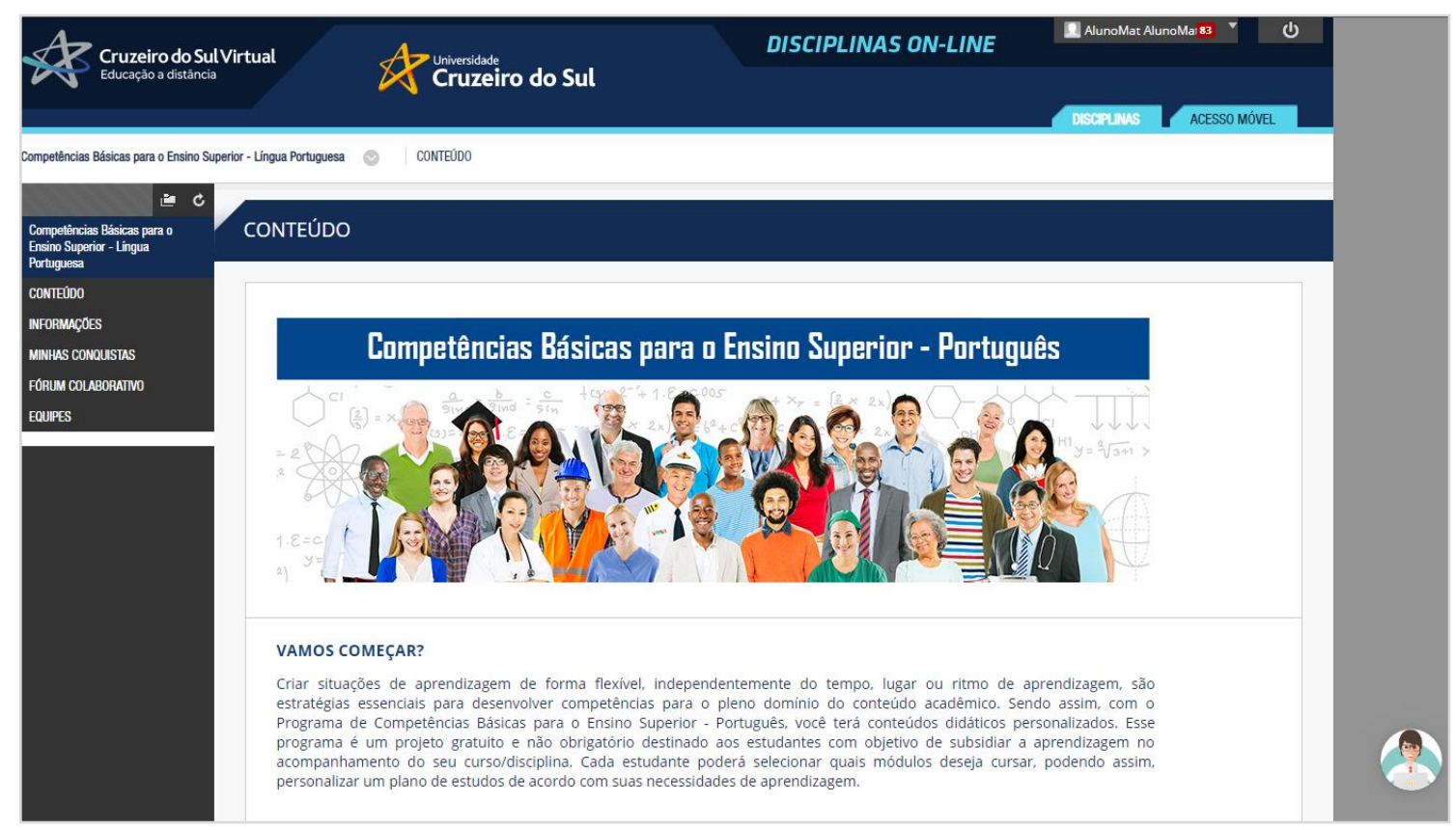

Figura 2. Modelo do curso de Competências Básicas Fonte: Ota (2018)

A partir do atendimento prestados aos alunos em fórum colaborativo e webinars, a equipe responsável pelo projeto, realizou a categorização das principais dúvidas e questionamento dos estudantes, dando origem a etapa 3, com o objetivo de aprimorar a oferta dos cursos. Nesta última etapa de aprimoramento, um total de 7593 estudantes estão em fase de conclusão dos cursos, sendo estes respectivamente: 4483 alunos realizando a formação de CBL LPO e 3110 em Matemática.

Uma ação ainda em andamento, foi a integração de um assistente virtual para ajudar os estudantes durante a experiência da trilha de aprendizagem. Zeki, Bot de interação acadêmica (Figura 3), criado em 2017 pelo Núcleo de Educação a Distância do Centro 
Universitário de João Pessoa foi incorporado aos cursos para responder as principais dúvidas, já mapeadas, aos novos estudantes.

Zeki foi concebido pelos princípios do retrieval based model, com a finalidade de interagir com uma acurácia de domínio fechado, isto é, o conhecimento é alimentado a partir de uma base de dados, incluíndo perguntas e possíveis respostas. Este tipo de Bot permite a implementação rápida, tendo em vista que se orienta a partir de regras (base de aprendizagem) predefinidas. A saber, a base de dados foi conectada à plataforma Dialogflow no sentido de permitir a construção da interface de conversação, suportado pelos elementos da inteligência artificial e o algoritmo de machine learning. Um ponto de fragilidade está no contato inicial com textos fora da sua base, requer, neste âmbito, uma direcionamento com estratégias supervisionadas com finalidade de identificar uma resposta viável ao questionamento e/ou à nova informação solicitada.

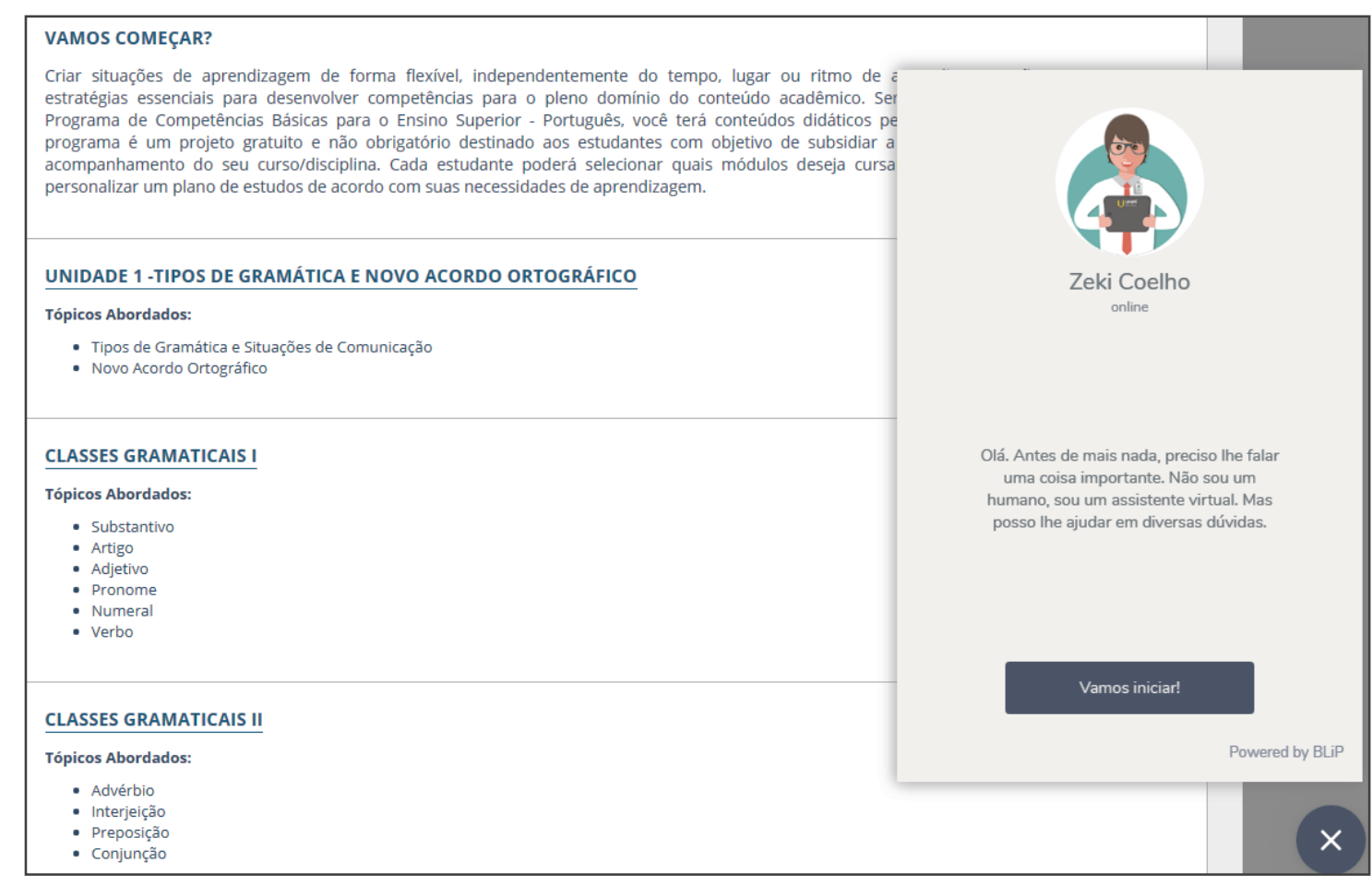

Figura 3. Integração do Assistente Virtual Fonte: Ota (2018)

Nos links: http://bit.ly/cbl portugues e http://bit.ly/cbl mat, o leitor pode ter acesso à versão digital do modelo proposto e realizar a interação, conferindo as questões de usabilidade aplicada no curso CBL.

\section{Considerações finais}

Em linhas gerais, a adoção de trilhas de aprendizagem em espaços virtuais de aprendizagem equipados de recursos e de flexibilização de integração, permite desenvolver modelos de cursos que vão ao encontro das necessidades, competências e pré-requisitos dos aprendizes. A intencionalidade pedagógica aplicada na escolha do objeto tecnológico, enriquece a experiência percorrida na navegação de um determinado curso e 
durante a proposição dos conteúdos, ao recorrer às metodologias ativas, por exemplo, traz à tona o engajamento dos estudantes, além de evidenciar aspectos de personalização da aprendizagem em caráter individual, da turma ou até mesmo do curso.

Ao adotar trilhas de aprendizagem que possuem estratégias adaptativas para personalização, tem-se também reconhecimento das características e necessidades individuais, fornecendo informações para a proposição de conteúdos. Adaptar-se às peculiaridades dos alunos faz também da educação a distância uma opção incontestável para assegurar a continuidade dos estudos e proporcionar um ensino de qualidade mesmo em áreas remotas. Um outro fator importante frente à massificação de cursos, é refletido em iniciativas de projetos e atividades que possam dar suporte às dificuldades dos estudantes. Os cursos ofertados de forma online precisam romper com suas estruturas enciclopédicas de modo a oportunizar que seus alunos possam ir além de receptores de informações para autores com capacidade de pensar criticamente as informações que recebem (OLIVEIRA e NAUROSKI, 2016, p.6).

O modelo dos dois cursos (CBL MAT e CBL LPO) compartilhados neste trabalho representa uma preocupação em nível institucional em gerar ações interventivas para desenvolver competências básicas de aprendizagem. Tal ação permite àqueles que tiveram uma formação inicial frágil e distante do esperado no ensino superior, acompanhar o curso escolhido.

Os resultados preliminares obtidos neste estudo demonstraram que as etapas de modelagem dos cursos, adicionadas a validação e feedback dos estudantes foram fundamentais para as práticas pedagógicas adotadas. Como dito, as possibilidades advindas da nova geração de ambientes virtuais são fatores que permitiram com o uso intencional da tecnologia, criar situações de aprendizagem que direcionam o olhar para as necessidades reais dos estudantes, seja em ordem acadêmica ou profissional. A integração de assistentes virtuais também se configura como uma ação inovadora, uma vez que são ampliadas formas de interação e mecanismos para estreitar a relação do aluno diante das competências esperadas.

Em suma, a contribuição desta investigação permite a reutilização do modelo desenvolvido para demais cursos/trilhas e até mesmo em outros ambientes virtuais. Como estudo futuro, espera-se que seja possível estender a oferta dos dois cursos para todos os estudantes, estimando um total de mais de 220 mil estudantes, além do aprofundamento de novos estudos que possam avaliar o impacto da utilização de assistentes virtuais em trilhas de aprendizagem.

\section{Referências}

ANDERSON, T., e SHATTUCK, J. Design-based research: A decade of progress in education research?. Educational researcher, 41(1), 16-25, 2012.

FONTE, F. A., RIAL, J. C. B., NISTAL, M. L.. Tq-bot: an aiml-based tutor and evaluator bot. Journal of Universal Computer Science, 15(7), 1486-1495, 2009.

GARRISON, R; ANDERSON,T. E-Learning in the 21st century. London: Routledge Falmer, 2003. 
GEMIGNANI, E. Y. M. Y. Formação de professores e metodologias ativas de ensinoaprendizagem: ensinar para a compreensão. (Vol.1) Recife: Fronteiras da Educação, 2013. GRAF, S., LIN, F., KINSHUK, A., e MCGREAL, R. Intelligent and Adaptive Learning Systems: Technology Enhanced Support for Learners and Teachers.IGI Global, 2012.

KATSIFLI, D. Reasons to care about Adaptive online learning. Disponível em: http://blog.blackboard.com/adaptive-online-learning/?lang=uk. Acesso 20.05.2019.

KEEFE, James W. Learning style: An overview. Student learning styles: Diagnosing and prescribing programs, v. 1, p. 1-17, 1979.

NICOLETE, P.C. Integração de tecnologia na educação: grupo de trabalho em experimentação remota móvel. (Dissertação de Mestrado não publicada). Universidade Federal de Santa Catarina, Araranguá, 2016.

OLIVEIRA; T.S. e NAUROSKI, E. Novas modalidades de ensino como forma de mudança social - análise do impacto do EAD no desenvolvimento regional. In XI Jornadas LatinoAmericanas de Estudos Sociais da Ciência e da Tecnologia (s.p.) Curitiba: ESOCITE 2016, 2016.

OLIVER, R. Exploring strategies for online teaching and learning. Distance Education Journal. 20:2, 1999. 240-254.

OTA, M.A. Adaptatividade em ambientes virtuais: uma proposta para personalizar a aprendizagem em cursos híbridos de ensino superior. (Tese de Doutorado não publicada). Universidade do Cruzeiro do Sul, São Paulo, 2018.

PRIMO, Alex et al. O uso de chatterbots na educação à distância. LEC/UFRGS Projeto: Rede Telemática para Formação de Educadores MEC/OEA. Porto Alegre, 2000.

RAMOS, D., Oliveira, E., MONTEVERDE, I., e OLIVEIRA, K. Trilhas de Aprendizagem em Ambientes Virtuais de Ensino-aprendizagem: Uma Revisão Sistemática da Literatura. In Anais do XXVI Simpósio Brasileiro de Informática na Educação (SBIE 2015), 2015. pp. 338-347.

REEVES, T.C. Design research from a technology perspective. In J. van den Akker, K. Gravemeijer, S. McKenney, \& N. Nieveen (Eds.), Educational design research (pp. 5266). London: Routledge, 2006.

REATEGUI, Eliseo; LORENZATTI, Alexandre. Um assistente virtual para resolução de dúvidas e recomendação de conteúdo. Encontro Nacional de Inteligência Artificial, 2005

RIST, Thomas et al. Adding animated presentation agents to the interface. In: Proceedings of the 2nd international conference on Intelligent user interfaces. ACM, 1997. p. 79-86.

SANTOS, Leila Maria Araújo. A inserção de um agente conversacional animado em um ambiente virtual de aprendizagem a partir da teoria da carga cognitiva. 2009.

SGANDERLA, Rachele Bianchi et al. BonoBOT: Um chatterbot para interação com usuários em um sistema tutor inteligente. In: Brazilian Symposium on Computers in Education (Simpósio Brasileiro de Informática na Educação-SBIE). 2003. p. 435-444. 
TAFNER, E. P et al. Trilhas de aprendizagem: uma nova concepção nos ambientes virtuais de aprendizagem-AVA. In Congresso Internacional de Educação a Distância (Vol. 18), 2012.

TIBOLA, L. et al. Virtual laboratory for promoting engagement and complex learning. In ELearn: World Conference on E-Learning in Corporate, Government, Healthcare, and Higher Education. Association for the Advancement of Computing in Education (AACE), 2014.

TRUONG, Huong May. Integrating learning styles and adaptive e-learning system: Current developments, problems and opportunities. Computers in human behavior, v. 55, p. 1185-1193, 2016.

U.S. Department of Education, Office of Educational Technology (2017). Reimagining the role of technology in education: 2017 national education technology plan update. Available at: https://tech.ed.gov/files/2017/01/NETP17.pdf (Accessed: 14 April 2018).

XIE, Haoran et al. Trends and development in technology-enhanced adaptive/personalized learning: A systematic review of journal publications from 2007 to 2017. Computers \& Education, p. 103599, 2019.

\section{Agradecimentos}

À equipe NEAD do Centro Universitário de João Pessoa (UNIPÊ), em especial, aos professores Luciano H. G. Almeida e Izabel Cavalcanti B. Lamenha pela contribuição no projeto $\mathrm{CBL}$ a partir da integração e autoria/criação do assistente virtual (Zeki).

Às Universidades UNIFRAN e UNICSUL pelo apoio e incentivo à pesquisa. 